\title{
EFICÁCIA ANTI-HELMÍNTICA DA IVERMECTINA EM EQUINOS: EXAMES COPROPARASITOLÓGICOS E HEMATOLÓGICOS
}

\section{ANTIHELMINTHIC EFFICACY OF IVERMECTIN IN HORSES: COPROPARASITOLOGICAL AND HEMATOLOGICAL EXAMS}

\author{
Fernando Cristino Barbosa ${ }^{1^{*}}$ \\ Wilson Junior Oliveira ${ }^{1}$ \\ Priscila Cristina Costa ${ }^{1}$ \\ Antonio Vicente Mundim ${ }^{1}$ \\ 1 Universidade Federal de Uberlândia, Uberlândia, MG, Brasil. \\ *Autor para correspondência - fcbarbosa@netsite.com.br
}

\section{Resumo}

O objetivo do presente estudo foi avaliar a eficácia da ivermectina a $2 \%$ em equinos, através de exames coproparasitológicos e hematológicos. $\mathrm{O}$ experimento foi realizado em uma fazenda no município de Uberlândia, MG. Foram utilizados 24 equinos, de três a oito anos, divididos em dois grupos, conforme valores dos OPG. Contagens de OPG e coproculturas foram realizadas nos dias D0, $\mathrm{D}+7$ e D+14. Os animais foram tratados com a formulação pasta a base de ivermectina $2 \%$, na dose de $200 \mathrm{mcg} / \mathrm{kg}$, via oral. Amostras de sangue foram coletadas nos dias D0, D+7 e D+14 para avaliação dos parâmetros hematológicos. A presença de ovos, característicos da família Strongylidae e identificação de larvas características de ciatostomíneos demonstrou o parasitismo dos animais por pequenos estrôngilos. Nos D+7 e D+14 houve a redução significativa do OPG no grupo tratado com eficácia de 99,5 e $99,7 \%$, respectivamente. Observou-se tendência do quadro eritrocitário manter-se próximo aos limites inferiores e discreta leucocitose com neutrófilos próximos dos valores superiores. Pode-se inferir que a ivermectina é eficaz contra os ciatostomíneos e alterações hematológicas significativas não foram observadas.

Palavras-chave: ciatostomíneos; equinos; nematódeos; OPG.

\begin{abstract}
The aim of the present study was to evaluate the efficacy of ivermectin at $2 \%$ in horses, through coproparasitological and hematological exams. The experiment was performed at a farm in the city of Uberlândia, Minas Gerais State. Twenty-four horses were used, ranging from 3 to 8 years of age, divided into 2 groups according to EPG values. EPG counts and coprocultures were performed on days D0, D+7 and D+14. The animals were treated with the ivermectin $2 \%$-based paste formulation at a dose of $200 \mathrm{mcg} / \mathrm{kg}$, orally. Blood samples were collected on days D0, D+7 and D+14 for evaluation of haematological parameters. The presence of eggs that are characteristic of the Strongylidae family and the identification of larvae that are characteristic of cyathostomins demonstrated the parasitism of the animals by small strongyles. At D+7 and D+14 there was a significant reduction of EPG in the treated group with an efficacy of 99.5 and $99.7 \%$, respectively. A
\end{abstract}


tendency of the erythrocyte framework to remain close to the lower limits was observed, besides a slight leukocytosis, with neutrophils close to the highest values. It can be inferred that ivermectin is effective against cyastostomines, and no significant haematological changes were observed.

Keywords: cyathostomins; EPG; horses; nematodes.

Recebido em: 12 dezembro de 2016

Aceito em: 11 dezembro de 2017

\section{Introdução}

A equinocultura vem se tornando um importante segmento do agronegócio brasileiro. O Brasil é o terceiro país com maior população de equinos, com cerca de 5.514.601 animais, e o estado de Minas Gerais é o mais populoso, com 763.780 cabeças $^{(1,2)}$.

Dentre todos os fatores que devem ser levados em consideração quando o assunto é a sanidade dos equinos, o parasitismo ocupa lugar de destaque devido aos prejuízos consequentes da infecção parasitária. Os helmintos podem causar desde um pequeno desconforto abdominal acompanhado ou não de fraqueza, pelagem áspera, retardo de crescimento, diminuição do desempenho, redução da digestão e absorção de nutrientes, hiporexia, anemia, diarreias ou constipações até episódios fulminantes de cólica e morte ${ }^{(3,4)}$.

Importante destacar que os equinos apresentam uma grande variedade de parasitos em sua fauna helmíntica e algumas espécies e gêneros tais como os pequenos estrôngilos ou ciatostomíneos e os grandes estrôngilos. Ainda, Parascaris equorum, Oxyuris equi, Strongyloides westeri, Trichostrongylus axei, Gasterophilus spp., Habronema spp., Anoplocephala spp. ${ }^{(5)}$ são de elevada importância.

Os grandes estrôngilos, durante o estágio larvar, migram em órgãos vitais, inclusive no sistema circulatório causando lesões de gravidade variada ${ }^{(6)}$. Os ciatostomíneos são considerados os helmintos de maior importância. Isso se dá pela sua atual prevalência, potencial patogênico, capacidade de resistência no meio ambiente, podendo sobreviver por longos períodos nas pastagens, e capacidade de desenvolver resistência anti-helmíntica ${ }^{(7,8)}$.

A administração de anti-helmíntico aos animais é um importante componente de um programa de controle e prevenção das infecções parasitárias. Dentre os compostos disponíveis, existem quatro grupos químicos distintos que são os mais utilizados: os benzimidazóis (ex.: albendazole, fenbendazole e oxibendazole), as pirimidinas (ex.: pamoato de pirantel) e o grupo das lactonas macrocíclicas (ex.: ivermectina, moxidectina, abamectina e doramectina) ${ }^{(5,9)}$.

Nas últimas décadas, o uso indiscriminado dessas drogas colaborou para o surgimento de populações de nematoides resistentes aos anti-helmínticos, principalmente os pertencentes à subfamília Cyathostominae, comumente conhecidos como ciatostomíneos, ameaçando seriamente a saúde, o bem-estar e a produção equina em diversas localidades do mundo ${ }^{(8,10-12)}$.

Levando em conta que a resistência à ivermectina vem se tornando comum entre os parasitos gastrintestinais dos pequenos ruminantes e bovinos, estudiosos sugerem que a resistência à ivermectina pelos ciatostomíneos será um fato inevitável ${ }^{(12-14)}$. 
Outro agravante é o fato de haver pouca perspectiva de surgimento de um novo grupo químico de anti-helmínticos para equinos ${ }^{(15)}$. Por isso, é muito importante o conhecimento da real situação da susceptibilidade das populações de nematódeos aos antiparasitários, dentro das diferentes características das propriedades.

A fim de determinar se um anti-helmíntico é eficiente ou não em uma determinada população de helmintos, o teste de resistência adequado para avaliar a eficácia de todas as classes de medicamentos disponíveis no mercado é o teste de redução da contagem de ovos nas fezes (TRCOF) ${ }^{(16-19)}$.

O presente estudo teve como objetivo avaliar a eficácia anti-helmíntica de uma formulação comercial à base de ivermectina $2 \%$, na apresentação pasta, administrada por via oral, em equinos naturalmente infectados, através de exames coproparasitológicos e hematológicos.

\section{Material e Métodos}

O experimento foi desenvolvido em uma fazenda no município de Uberlândia-MG. Foram utilizados 24 equinos, de ambos os sexos de dois a oito anos de idade, naturalmente infectados por helmintos. O protocolo foi aprovado pela Comissão de Ética na Utilização de Animais (CEUA/UFU), com o registro $n^{\circ} 113 / 16$.

No dia 3, antes do tratamento (D-3), os animais foram identificados e submetidos à coleta de fezes diretamente da ampola retal, em sacos plásticos descartáveis, identificados individualmente, acondicionadas em gelo reciclável e transportadas até o Laboratório de Doenças Parasitárias da Faculdade de Medicina Veterinária da Universidade Federal de Uberlândia (FAMEV-UFU). Para a contagem de ovos nas fezes (OPG), utilizou-se a técnica de Gordon e Whitloch ${ }^{(20)}$ modificada, com a detecção mínima de 50 OPG, e randomização dos grupos.

Os animais foram divididos em dois grupos experimentais com 12 animais cada, conforme os valores dos OPG. Os dois animais com contagem mais elevada foram destinados à repetição número 1, os dois seguintes à repetição número 2 , até que se formaram 12 repetições. Após a distribuição, foram sorteados aleatoriamente os grupos, de acordo com o tratamento proposto: Grupo T (Tratado) - 12 animais tratados com a formulação pasta à base de ivermectina $2 \%$, com seringa dosadora específica na dose de 1,0g/100 kg de PV (200 mcg/kg), via oral. Grupo C (Controle) - 12 animais que permaneceram sem tratamento.

A análise parasitológica permitiu a seleção de animais apresentando maiores níveis de infecção por nematoides, com valores mínimos e máximos de 800 a 2150 OPG, respectivamente, e média de 1635 \pm 365 OPG. Após a randomização dos grupos, o grupo tratado (GT) ficou com OPG médio de 1608 \pm 327 e o grupo controle $(\mathrm{GC}) 1663 \pm 413$.

Para avaliar a eficácia do produto, foi usado o teste de redução na contagem de ovos por grama de fezes (TRCOF), utilizando a fórmula descrita por Coles et al. ${ }^{(21)}$ : \% eficácia $=[1-(\mathrm{OPGt} / \mathrm{OPGc})] \mathrm{x}$ 100.

As amostras de fezes foram coletadas no dia do tratamento (D0) e nos dias D+7 e D+14 póstratamento para quantificação do OPG e coprocultura. As coproculturas foram realizadas com um pool de fezes de cada grupo, segundo Roberts e Sullivan ${ }^{(22)}$, para obtenção das larvas e posterior 
identificação dos nematódeos da subfamília Strongylinae e Cyatosthominae, de acordo com a chave dicotômica proposta por Madeira de Carvalho et al. ${ }^{(23,24)}$

Foram coletadas amostras de sangue dos 24 animais para a realização de hemogramas, visando avaliar os parâmetros hematológicos no dia do tratamento (D0) e nos dias D+7 e D+14 pós-tratamento. Coletou-se de cada animal $2 \mathrm{~mL}$ de sangue, por venopunção da jugular em tubos de coleta (Vacutainer ${ }^{\circledR}$ ) contendo 0,1 mL de EDTA K3 (ácido etilenodiaminotetracético sal tripotássico) em solução a $10 \%$ para realização dos hemogramas em analisador automático $\mathrm{ABC} \mathrm{Vet}^{\mathrm{TM}}$. Os seguintes parâmetros foram analisados: volume globular (VG), hematimetria $(\mathrm{He})$, hemoglobinometria $(\mathrm{Hb})$, volume corpuscular médio (VCM), hemoglobina corpuscular média (HCM), concentração de hemoglobina corpuscular média (CHCM) e leucometria.

A contagem diferencial de leucócitos foi realizada em extensões sanguíneas coradas pelo método May-Grüwald-Giemsa, nas quais foram identificadas e contadas 100 células e estabelecida a fórmula leucocitária relativa ${ }^{(25)}$.

$\mathrm{Na}$ análise estatística, foram utilizados os softwares Excel 2016 e BioEstat 5.0 para execução dos cálculos. A verificação da normalidade dos dados foi realizada pelo teste de Shapiro-Wilk. Para os dados dos OPG e parâmetros hematológicos, que apresentaram distribuição normal, foram utilizados dois testes de hipóteses, o teste de F e o teste de $t$ de Student. Para os dados que não apresentaram distribuição normal foi utilizado o teste de Mann-Whitney.

\section{Resultados}

$\mathrm{Na}$ análise parasitológica, foram observados ovos morulados característicos de parasitos da família Strongylidae, segundo Hoffmann ${ }^{(26)}$. O resultado da análise quantitativa de ovos por grama de fezes $(\mathrm{OPG})$ no dia do tratamento (D0) foi semelhante $(\mathrm{p}>0,05)$ (Tabela 1).

Tabela 1 - Valores médios, desvio padrão, valores mínimos e máximos do OPG de equinos do grupo tratado com uma formulação oral de Ivermectina $2 \%$ e grupo controle, Uberlândia - MG

\begin{tabular}{|c|c|c|c|c|c|c|}
\hline \multirow{3}{*}{ OPG } & \multicolumn{6}{|c|}{ Momentos (dias) } \\
\hline & \multicolumn{3}{|c|}{ Grupo tratado } & \multicolumn{3}{|c|}{ Grupo controle } \\
\hline & D0 & $\mathbf{D}+7$ & D+14 & D0 & $\mathbf{D}+7$ & $\mathrm{D}+14$ \\
\hline Média & $1521 \mathrm{~A}$ & $8 \mathrm{~B}$ & 4B & $1550 \mathrm{~A}$ & $1642 \mathrm{~A}$ & $1600 \mathrm{~A}$ \\
\hline DP & 224 & 29 & 14 & 382 & 480 & 394 \\
\hline Máximo & 1800 & 100 & 50 & 2150 & 2150 & 2050 \\
\hline Mínimo & 1150 & 0 & 0 & 700 & 600 & 700 \\
\hline \% Eficácia & - & 99,5 & 99,7 & - & - & - \\
\hline
\end{tabular}

Letras maiúsculas diferentes nas linhas representam valores significativamente diferentes $(\mathrm{p}<0,05)$ entre os grupos, nos diversos momentos

Nos D+7 e D+14, houve a redução significativa do OPG no grupo tratado, com a média de $8 \pm 29$ e $4 \pm 14$, respectivamente, com apenas um animal positivo, apresentando diferença significativa com o grupo tratado $(\mathrm{p}<0,05)$, as taxas de eficácias nos dias D+7 e D+14 foram de 99,5 e 99,7\%, respectivamente. 
A identificação das larvas infectantes obtidas das coproculturas, realizadas antes e após tratamento, em ambos os grupos, possibilitou identificar larvas L3 com características morfológicas da Subfamília Cyathostominae, baseada na observação do número e forma das células intestinais, comprimento total da larva, presença ou ausência de bainha e aspecto da bainha da cauda ${ }^{(23,24)}$.

Observou-se que, durante o período de avaliação, o volume globular (VG) e a hemoglobina (Hb) se mantiveram abaixo dos limites mínimos de referência ${ }^{(27)}$, não apresentando diferença significativa entre os grupos ( $>>0,05)$, exceto o $\mathrm{VG}$ e número de hemácias no dia $\mathrm{D}+14$, no grupo tratado, que foram superiores ao grupo controle $(\mathrm{P}<0,05)$ (Tabela 2$)$.

Tabela 2 - Valores médios e desvio padrão dos parâmetros do eritrograma de equinos do grupo tratado (GT) com uma formulação oral de ivermectina $2 \%$ e grupo controle (GC), Uberlândia MG

\begin{tabular}{|c|c|c|c|c|c|}
\hline \multirow{2}{*}{ Parâmetros } & \multicolumn{4}{|c|}{ Momentos (dias) } & \multirow{2}{*}{$\begin{array}{c}\text { Referência } \\
1\end{array}$} \\
\hline & Grupos & D0 & $\mathbf{D}+7$ & $\mathrm{D}+14$ & \\
\hline \multirow{2}{*}{$\begin{array}{l}\text { Hemáceas } \\
\left(\mathrm{x} 10^{6} / \mu \mathrm{L}\right)\end{array}$} & GC & $7,3 \pm 0,6$ & $7,3 \pm 0,4$ & $7,2 \pm 0,6 b$ & \multirow{2}{*}{$6,8-12,9$} \\
\hline & GT & $7,4 \pm 0,6$ & $7,3 \pm 0,6$ & $7,8 \pm 0,7 \mathrm{a}$ & \\
\hline \multirow{2}{*}{$\begin{array}{c}\text { Hemoglobina } \\
\text { (g/dL) }\end{array}$} & GC & $10,3=0,7$ & $10,3 \pm 0,82$ & $10,1=0,8$ & \multirow{2}{*}{$11-19$} \\
\hline & GT & $10,4=0,7$ & $10,4 \pm 0,9$ & $10,7 \pm 1,1$ & \\
\hline \multirow{2}{*}{$\begin{array}{c}\text { Volume } \\
\text { Globular (\%) }\end{array}$} & $\mathrm{GC}$ & $29,7 \pm 2,3$ & $29,2 \pm 2,2$ & $29,2 \pm 1,9 b$ & \multirow{2}{*}{$32-53$} \\
\hline & GT & $30,1 \pm 2,6$ & $31,0 \pm 3,2$ & $32,1 \pm 4,1 \mathrm{a}$ & \\
\hline \multirow{2}{*}{ VCM (fL) } & $\mathrm{GC}$ & $40,5 \pm 2,3$ & $39,8 \pm 2,0 b$ & $40,7 \pm 2,6$ & \multirow{2}{*}{$37-58,5$} \\
\hline & GT & $41,0=2,9$ & $42,5=4,2 a$ & $41,4=4,1$ & \\
\hline \multirow{2}{*}{ HCM (pg) } & $\mathrm{GC}$ & $14,1 \pm 0,3$ & $14,0 \pm 0,5$ & $13,9 \pm 0,5$ & \multirow{2}{*}{$12,3-19,9$} \\
\hline & GT & $14,2 \pm 0,6$ & $14,3 \pm 1,0$ & $13,8 \pm 1,0$ & \\
\hline \multirow{2}{*}{$\begin{array}{l}\text { CHCM } \\
(\mathrm{g} / \mathrm{dL})\end{array}$} & $\mathrm{GC}$ & $35,0 \pm 1,3$ & $35,3 \pm 2,7$ & $34,2 \pm 1,9$ & \multirow{2}{*}{$31-38,6$} \\
\hline & GT & $34,8 \pm 2,4$ & $33,8 \pm 1,4$ & $33,6 \pm 2,4$ & \\
\hline
\end{tabular}

Letras minúsculas diferentes nas colunas representam valores significativamente diferentes $(p<0,05)$ entre 03 grupos, para cada parâmetro, nos diferentes momentos. ${ }^{1}$ - Orsine, Divers ${ }^{(27)}$

$\mathrm{Na}$ avaliação do leucograma, os leucócitos estiveram ligeiramente aumentados no grupo tratado, no dia D0, acompanhado pelos neutrófilos jovens (bastonetes) que permaneceram acima dos valores fisiológicos durante todos os momentos, em ambos os grupos, mas não apresentando diferença significativa entre eles $(\mathrm{p}>0,05)$ (Tabela 3$)$. 
Tabela 3 - Valores médios e DP dos parâmetros do leucograma de equinos do grupo tratado (GT) com uma formulação oral de Ivermectina $2 \%$ e grupo controle (GC), Uberlândia - MG

\begin{tabular}{|c|c|c|c|c|c|}
\hline \multirow{2}{*}{ Parâmetros } & \multirow{2}{*}{ Grupos } & \multicolumn{3}{|c|}{ Momentos (dias) } & \multirow{2}{*}{ Referência } \\
\hline & & D0 & $\mathrm{D}+7$ & $D+14$ & \\
\hline \multirow{2}{*}{$\begin{array}{c}\text { Leucócitos } \\
\left(\mathrm{x} 10^{3} / \mu \mathrm{L}\right)\end{array}$} & GC & $12,8 \pm 2,0$ & $13,6 \pm 2,6$ & $14,1 \pm 5,4$ & \multirow{2}{*}{$5,4-14,3$} \\
\hline & GT & $14,5 \pm 3,7$ & $14,0 \pm 3,6$ & $13,7 \pm 4,3$ & \\
\hline \multirow{2}{*}{$\begin{array}{c}\text { Bastonetes } \\
\left(\mathrm{x} 10^{3} / \mu \mathrm{L}\right)\end{array}$} & GC & $0,30 \pm 0,2$ & $0,43 \pm 0,2$ & $0,57 \pm 0,4$ & \multirow{2}{*}{$0-0,1$} \\
\hline & GT & $0,26 \pm 0,2$ & $0,64 \pm 0,3$ & $0,54 \pm 0,4$ & \\
\hline \multirow{2}{*}{$\begin{array}{l}\text { Segmentados } \\
\left(\times 10^{3} / \mu \mathrm{L}\right)\end{array}$} & $\mathrm{GC}$ & $6,9 \pm 1,7$ & $6,9 \pm 2,0$ & $7,3 \pm 3,9$ & \multirow{2}{*}{$2,26-8,58$} \\
\hline & GT & $7,7 \pm 2,3$ & $6,4 \pm 1,9$ & $6,9 \pm 2,7$ & \\
\hline \multirow{2}{*}{$\begin{array}{c}\text { Neutrófilos } \\
\text { Total }\left(x 10^{3} / \mu \mathrm{L}\right)\end{array}$} & $\mathrm{GC}$ & $7,2 \pm 1,8$ & $7,4 \pm 2,0$ & $7,8 \pm 4,1$ & \multirow{2}{*}{$2,3-8,6$} \\
\hline & GT & $8,0 \pm 2,3$ & $7,0 \pm 2,0$ & $7,4 \pm 3,1$ & \\
\hline \multirow{2}{*}{$\begin{array}{c}\text { Eosinófilos } \\
\left(\mathrm{x} 10^{3} / \mu \mathrm{L}\right)\end{array}$} & GC & $0,7 \pm 0,6$ & $0,7 \pm 0,3$ & $0,7 \pm 0,4$ & \multirow{2}{*}{$0-1$} \\
\hline & GT & $0,6 \pm 0,3$ & $0,8 \pm 0,4$ & $0,7 \pm 0,4$ & \\
\hline \multirow{2}{*}{$\begin{array}{l}\text { Basófilos } \\
\left(\mathrm{x} 10^{3} / \mu \mathrm{L}\right)\end{array}$} & $\mathrm{GC}$ & $0,05 \pm 0,08$ & $0,07 \pm 0,1$ & $0,1 \pm 0,1$ & \multirow{2}{*}{$0-0,29$} \\
\hline & GT & $0,07 \pm 0,1$ & $0,1 \pm 0,2$ & $0,1=0,1$ & \\
\hline \multirow{2}{*}{$\begin{array}{l}\text { Monócitos } \\
\left(\times 10^{3} / \mu \mathrm{L}\right)\end{array}$} & GC & $0,4 \pm 0,2$ & $0,7 \pm 0,3$ & $0,7 \pm 0,3$ & \multirow{2}{*}{$0-1$} \\
\hline & GT & $0,4 \pm 0,2$ & $0,9 \pm 0,4$ & $0,7 \pm 0,3$ & \\
\hline \multirow{2}{*}{$\begin{array}{l}\text { Linfócitos } \\
\left(\times 10^{3} / \mu \mathrm{L}\right)\end{array}$} & GC & $4,3 \pm 0,9$ & $4,6 \pm 1,3$ & $4,6 \pm 1,1$ & \multirow{2}{*}{$1,5-7,7$} \\
\hline & GT & $5,3 \pm 1,9$ & $5,0 \pm 1,9$ & $4,6 \pm 1,8$ & \\
\hline
\end{tabular}

\section{Discussão}

Apesar de os valores de OPG não serem um retrato fiel do número de parasitos presentes no hospedeiro $^{(28)}$, os resultados obtidos neste trabalho sugerem que os animais estavam com uma elevada infecção por formas adultas de estrongilídeos. Considerando que uma contagem de 200 OPG já é um indicativo de infecção com necessidade de tratamento anti-helmíntico ${ }^{(29)}$, o grau de parasitismo dos animais foi considerado adequado para a realização do estudo.

A presença de ovos morulados característicos da família Strongylidae e a identificação de larvas L3 características de ciatostomíneos demonstram a predominância da infecção por pequenos estrôngilos nos equinos avaliados. Resultados semelhantes aos de Dornbusch et al. ${ }^{(30)}$ que observaram somente larvas de ciatostomíneos nas coproculturas de equinos da raça crioula mantidos a pasto. Ferreira et al. ${ }^{(31)}$ também observaram maior prevalência de parasitas da subfamília Cyathostominae do que da 
subfamília Strongylinae. Muitas vezes, os ciatostomíneos compreendem 95-100\% da carga parasitária total nos equinos ${ }^{(5,32)}$.

Estudos demonstram que, antes da utilização das lactonas macrocíclicas no controle parasitário em equinos, a prevalência dos grandes estrôngilos, principalmente do S. vulgaris, era de $80 \%$ a $100 \%{ }^{(33)}$. Essa prevalência diminuiu de maneira drástica no decorrer dos anos de utilização desses compostos anti-helmínticos ${ }^{(14,34)}$, contribuindo para o predomínio e consequente resistência dos ciatostomíneos. Isso, possivelmente, justifica a infecção dos equinos do presente estudo pelos ciatostomíneos, pois, no controle parasitário dessa criação, utiliza-se com certa frequência os anti-helmínticos do grupo das lactonas macrocíclicas.

No teste de redução da contagem de ovos nas fezes (TRCOF), os valores de OPG individuais devem ser transformados em médias do grupo para que os dados se aproximem de uma distribuição normal. Usando esse método, o ponto de corte para determinar a eficácia anti-helmíntica é de 95\% para lactonas macrocíclicas e $90 \%$ para benzimidazóis e pamoato de pirantel ${ }^{(35)}$.

Adotando esse critério no presente estudo, os valores médios do OPG do grupo tratado nos dias D+7 e $\mathrm{D}+14$ foram significativamente menores $(\mathrm{p}<0,05)$ que os do grupo controle, com eficácias de 99,5 e $99,7 \%$, respectivamente (Tabela1); demonstrando a ação anti-helmíntica da ivermectina nos estágios adultos dos parasitos.

No grupo controle, não houve diferença $(p>0,05)$ nos diferentes momentos de avaliação, demonstrando que a infecção se manteve inalterada durante todo o estudo, sugerindo o equilíbrio na relação hospedeiro-parasito.

Embora existam relatos de resistência dos ciatostomíneos à ivermectina ${ }^{(13,14,36)}$, os resultados do presente estudo com equinos naturalmente infectados demonstram a eficácia da ivermectina avaliada pelo teste de redução da contagem de ovos nas fezes, conforme relatado por outros autores ${ }^{(37-41)}$.

Um dos principais motivos para a demora no desenvolvimento da resistência a este composto pode ser explicado pelo fato de que ele não tem ação sobre as larvas de quarto estágio encistadas na mucosa intestinal, que é considerada uma grande população de refúgia ${ }^{(42)}$.

Portanto, os anti-helmínticos devem ser selecionados de acordo com a sua eficiência na redução dos nematoides gastrintestinais dos equídeos, em especial os ciatostomíneos, que, na maioria das vezes, predominam na infecção parasitária e são os principais responsáveis pela resistência aos antihelmínticos.

A hematimetria demonstrou que o número de eritrócitos se manteve próximo aos limites inferiores de normalidade em todos os momentos em ambos os grupos. No entanto, no dia D+14, o valor encontrado para o grupo tratado foi superior ao controle $(\mathrm{p}<0,05)$, acompanhando a recuperação do volume globular, que ficou dentro da normalidade para a espécie e maior que o grupo controle $(\mathrm{p}<0,05)$. A tendência à anemia nos animais desta pesquisa possivelmente pode estar relacionada ao parasitismo apresentado por eles, embora não apresentassem sintomas clínicos de verminose, pois, no D+14 pós-tratamento, observou-se no grupo tratado o retorno do volume globular aos parâmetros fisiológicos para a espécie.

Esses achados corroboram os encontrados por Reichmann et al. ${ }^{(43)}$ e Lhamas et al. ${ }^{(44)}$, que relatam a tendência à ocorrência de anemia e divergem dos encontrados por Sartori Filho et al. ${ }^{(45)}$, que não observaram alterações hematológicas associadas à infecção natural por estrongilídeos. 
O quadro leucocitário revelou discreta leucocitose, no dia D0, no grupo tratado, com neutrófilos próximos dos valores superiores e desvio nuclear de neutrófilos para a esquerda (DNNE). Achados que se assemelham aos de Reichmann et al. ${ }^{(43)}$ e Lhamas et al. ${ }^{(44)}$, que observaram tendência à leucocitose por neutrofilia e valores normais de linfócitos e eosinófilos. Não foi observada diferença ( $>>0,05)$ nos valores encontrados para os leucócitos, entre os grupos, em todos os momentos. No entanto, observou-se a redução do número de leucócitos no dia D+14, nos animais tratados, com valor médio dentro dos limites da normalidade e menor que o grupo controle.

A eosinofilia não foi constatada nos animais estudados, contradizendo em parte o conceito de que a mesma deva acompanhar os quadros de endoparasitismo em equinos.

Infecções experimentais maciças puras por $S$. vulgaris geraram quadro de eosinofilia como consequência da migração larval dos parasitos ${ }^{(46)}$. No presente estudo, a infecção parasitária dos animais foi por ciatostomíneos e estes não apresentam o mesmo tipo de migração dos grandes estrôngilos. Esses achados reforçam a hipótese de que a carga parasitária e a espécie dos parasitos a que esses animais estavam submetidos não eram suficientes para alterar de forma significativa os parâmetros do leucograma.

As variações de resultados possivelmente decorrem das diferentes respostas imunológicas relacionadas com a carga parasitária, parasitos envolvidos e as condições clínicas dos hospedeiros.

Os outros parâmetros leucocitários mantiveram-se dentro dos intervalos de referência, não havendo diferenças entre os grupos ( $p>0,05)$. Esses resultados são semelhantes aos observados por Sartori Filho et al. ${ }^{(4)}$ e Lhamas et al. ${ }^{(44)}$, que não encontraram diferenças no leucograma entre animais parasitados e após tratamento com anti-helmíntico.

\section{Conclusão}

Os resultados deste estudo permitem inferir que a ivermectina a $2 \%$ promove o controle eficaz dos ciatostomíneos. Observou-se também que os parâmetros hematológicos avaliados não tiveram alterações significativas relacionadas ao parasitismo.

\section{Referências}

1. Instituto Brasileiro de Geografia e Estatística - IBGE. Produção da pecuária estadual. Disponível em: http://www.ibge.gov.br/estadosat/temas.php?sigla=mg\&tema=pecuaria2014. Acesso em: 26 set. 2016.

2. Instituto Brasileiro de Geografia e Estatística - IBGE. Disponível em: http://seriesestatisticas.ibge.gov.br/series.aspx?vcodigo=PPM01 Acesso em: 26 set. 2016.

3. Brady HA, Nichols WT. Drug resistance in equine parasites: an emerging global problem. Journal of Equine Veterinary Science. 2009;29(5):285-295. Disponível em http://dx.doi.org/10.1016/j.jevs.2009.04.186. Acesso em: 29 de setembro de 2016.

4. Lagaggio VRA, Jorge LL, Oliveira V, Flores ML, Silva JH. Achados de formas parasitárias em camas de equinos. Santa Maria: $\quad$ [s.n.], 2007. Disponível em: http://www.hipismobrasil.com.br/teses/formas_parasitarias.asp. Acesso em: 8 de setembro de 2016. 
5. Molento MB. Resistência parasitária em helmintos de equinos e propostas de manejo. Ciência Rural. 2005;35(6): 1469-1477. Disponível em: http://www.scielo.br/pdf/cr/v35n6/a41v35n6.pdf. Acesso em: 26 de setembro de 2016.

6. Drudge JH, Lyons ET. Large Strongyles: Recent Advances. Veterinary Clinics of North America: Equine Pratice. 1986,2(2):263-280. Disponível em: https://doi.org/10.1016/S0749-0739(17)30716-2. Acesso em: 29 de agosto de 2016.

7. Lester HE, Spanton J, Stratford CH, Bartley DJ, Morgan ER, Hodgkinson JE, Coumbe K, Mair T, Swan B, Lemon G, Cookson R, Matthews JB. Anthelmintic efficacy against cyathostomins in horses in Southern England. Veterinary Parasitology. 2014;197(1-2):189-196. Disponível em: http://dx.doi.org/10.1016/j.vetpar.2013.06.009. Acesso em: 03 de outubro de 2016.

8. Peregrine AS, Molento MB, Kaplan RM, Nielsen, MK. Anthelmintic resistance in important parasites of horses: does it really matter? Veterinary Parasitology. 2014;201:1-8. Disponível em: http://dx.doi.org/10.1016/j.vetpar.2014.01.004. Acesso em: 8 de setembro de 2016.

9. Samson-Himmelstjerna GV. Anthelmintic resistance in equine parasites - detection, potential clinical relevance and implications for control. Veterinary Parasitology. 2012;185(1):2-8. Disponível em: http://dx.doi.org/10.1016/j.vetpar.2011.10.010. Acesso em: 20 de setembro de 2016.

10. Traversa D, Iorio R, Otranto D, Giangaspero A, Milillo P, Klei TR. Species-specific identification of equine cyathostomes resistant to fenbendazole and susceptible to oxibendazole and moxidectin by macroarray probing. Experimental Parasitology. 2009;121:92-95. Disponível em: https://doi.org/10.1016/j.exppara.2008.10.001. Acesso em: 25 de setembro de 2016.

11. Matthews JB. The future of helminth control in horses. Equine Veterinary Journal. 2014;46(1):10-11. Disponível em: http://dx.doi.org/10.1111/evj.12200. Acesso em: 03 de outubro de 2016.

12. Kaplan RM, Klei TR, Lyons ET, Lester G, Courtney CH, French DD, Tolliver SC, Vidyashankar AN, Zhao Y. Prevalence of anthelmintic resistant cyathostomes on horse farms. Journal of the American Veterinary Medical Association, Schaumburg. 2004;225(6):903-910. Disponível em: https://doi.org/10.2460/javma.2004.225.903. Acesso em: 29 de agosto de 2016.

13. Molento MB, Antunes J, Bentes RN, Coles GC. Anthelmintic resistant nematodes in Brazilian horses. The Veterinary Record, London. 2008;162(12):384-385. Disponível em: http://dx.doi.org/10.1136/vr.162.12.384. Acesso em: 31 de agosto de 2016.

14. Canever RJ, Bragab PRC, Boeckhc A, Grycajucka M, Biera D, Molento MB. Lack of Cyathostomin sp. reduction after anthelmintic treatment in horses in Brazil. Veterinary Parasitology, Amsterdam. 2013;194(1):35-39. Disponível em: https://doi.org/10.1016/j.vetpar.2012.12.020._Acesso em: 25 de setembro de 2016.

15. Nielsen MK, Kaplan RM, Thamsborg SM, Mon-Rad J, Olsen SN. Climatic influences on development and survival of free-living stages of equine strongyles: Implications for worm control strategies and managing anthelmintic resistance. The Veterinary Journal. 2007;174(1):23-32. Disponível em: https://doi.org/10.1016/j.tvj1.2006.05.009. Acesso em: 31 de agosto de 2016.

16. Coles GC, Jackson F, Pomroy WE, Prichard RK, Von Samson-Himmelstjerna G, Silvestre A, Taylor MA, Vercruysse J. The detection of anthelmintic resistance in nematodes of veterinary importance. Veterinary Parasitology, Amsterdam. 2006;136(3/4):167-185. Disponível em: http://dx.doi.org/10.1016/j.vetpar.2005.11.019. Acesso em: 25 de setembro de 2016.

17. Matthews JB, McArthur C, Robinson A, Jackson F. The in vitro diagnosis of anthelmintic resistance in cyathostomins. Veterinary Parasitology, Amsterdam. 2012;185(1):25-31. Disponível em: 
https://doi.org/10.1016/j.vetpar.2011.10.014. Acesso em: 8 de setembro de 2016.

18. Kaplan RM. Biological considerations in evaluating drug efficacy and resistance in equine strongyle parasites using fecal egg count data. 2008. In: Proceedings International Equine Parasite Drug Resistance Workshop. University of Copenhagen, Denmark.p.14-15.

19. Von Samson-Himmelstjerna G. Anthelmintic resistance in equine parasites - detection, potential clinical relevance and implications for control. Veterinary Parasitology. Amsterdam. 2012;185(1):2-8. Disponível em: https://doi.org/10.1016/j.vetpar.2011.10.010. Acesso em: 31 de agosto de 2016.

20. Gordon HMcL, Whitlock, HV. A new technique for counting nematode eggs in sheep faeces. Journal of the Council for Scientific and Industrial Research. 1939;12(1):50-52. Disponível em https://publications.csiro.au/rpr/download?pid=procite:21259a33-8a8e-4add-9315-f8338091a3e6\&dsid=DS1.

Acesso em: 31 de agosto de 2016.

21. Coles G, Bauer C, Borgsteede F, Geerts, S, Klei, TR., Taylor M, Waller P. World Association for the Advancement of Veterinary Parasitology (WAAVP) methods for the detection of anthelmintic resistance in nematodes of veterinary importance. Veterinary Parasitology. Amsterdam. 1992;44(1-2): 35-44. Disponível em: https://doi.org/10.1016/0304-4017(92)90141-U. Acesso em: 25 de agosto de 2016.

22. Roberts FHS, O'sullivan PJ. Methods for egg counts and larval cultures for strongyles infecting the gastrointestinal tract of cattle. Australian Journal of Agricultural Research. 1950;1(1):99-102. Disponível em: https://doi.org/10.1071/AR9500099.

23. Madeira de Carvalho LM, Fazendeiro MI, Afonso-Roque MM. Estudo morfométrico das larvas infectantes (L3) dos estrongilí́deos (Nematoda: Strongylidae) dos equídeos - 1. Género Cyathostomum s.l. Acta Parasitológica Portuguesa. 2004;11(1-2):23-32. Disponível em: https://www.researchgate.net/publication/273259568_ESTUDO_MORFOMETRICO_DAS_LARVAS_INF ECTANTES_L3_DOS_ESTRONGILIDEOS_NEMATODA_STRONGYLIDAE_DOS_EQUIDEOS_1_Genero_Cyathostomum_sl. Acesso em: 8 de setembro de 2016.

24. Madeira de Carvalho LM, Fazendeiro, MI, Afonso-Roque MM. Estudo morfométrico das larvas infectantes (L3) dos estrongilídeos (Nematoda: Strongylidae) dos equídeos. 4. Estudo das populações de ciatostomíneos de equídeos bravios e domésticos através do método de análise dos morfotipos de L3 de Cyathostomum sensu latum. Acta Parasitológica Portuguesa. 2008a;15(1-2):65-70. Disponível em: https://www.researchgate.net/publication/273259732. Acesso em: 8 de setembro de 2016.

25. Ferreira Neto JM, Viana ES, Magalhães LM. Patologia Clínica Veterinária. 1982, Rabelo, Belo Horizonte.

26. Hoffmann RP. Diagnóstico Parasitismo Veterinário. Sulina: Porto Alegre, 1987. 156p.

27. Orsine JA, Divers TJ. Equine Emergencies: Treatment and Procedures. $4^{\mathrm{a}}$ ed. St. Louis, Saunders. 2014. 861 p. ISBN-13: 978-1455708925

28. Uhlinger CA. Use of fecal egg count data in equine practice. Compendium on Continuing Education for the Practicing Veterinarian. 1993;15(5):742-748. Disponível em: https://geoscience.net/research/002/541/002541078.php\#close. Acesso em: 25 de agosto de 2016.

29. Pérez R, Godoy C, Palma C, Cabezas I, Muñoz L, Rubilar L, Arboix M, Alvinerie M. Plasma profiles of ivermectin in horses following oral or intramuscular administration. Jounal of Veterinary Medicine. 2003;50(6):297-302. Disponível em: http://dx.doi.org/10.1046/j.1439-0442.2003.00531.x. Acesso em: 8 de setembro de 2016.

30. Dornbusch PT, Micheloto PV, Santarém VA, De Carli LM, Camargo CE, Biava JS. Eficácia antihelmíntica da ivermectina "Pour-on" comparada com a formulação oral em gel nos equinos. Revista Acadêmica: Ciência Animal, Curitiba. 2006;4(4):21-24. Disponível em: 
http://dx.doi.org/10.7213/cienciaanimal.v4i4.9474. Acesso em: 8 de setembro de 2016.

31. Ferreira GMS, Dutra FAF, Filho EFA, Santos ACG. Parasitismo gastrintestinal e hematologia em equinos e asininos da mesorregião da aglomeração urbana, São Luís, Maranhão. Archives of Veterinary Science. 2014;19(2):22-30. Disponível em: http://dx.doi.org/10.5380/avs.v19i2.32898. Acesso em: 30 de agosto de 2016.

32. Gasser RB, Williamson RMC, Beveridge I. Anoplocephala perfoliata of horses - significant scope for further research improved diagnosis and control. Parasitology. 2005;131(1):1-13. Disponível em: https://doi.org/10.1017/S0031182004007127. Acesso em: 28 de agosto de 2016.

33. Slocombe JOD, Coté JF, Gannes RVG. The persistence of benzimidazole-resistant cyathostomes on horse farms in Ontario over 10 years and the effectiveness of ivermectin and moxidectin against these resistant strains. Canadian Veterinary Journal. 2008; 49:56-60. Disponível em https://www.ncbi.nlm.nih.gov/pmc/articles/PMC2147695/. Acesso em: 25 de agosto de 2016.

34. Madeira de Carvalho LM. In Memoriam Prof. Ignacio Navarrete López-Cózar, Edition: 1st, Chapter: Estrongilidose dos Equídeos - Biologia, Patologia, Epidemiologia e Controlo, Publisher: Universidad de Extremadura, Facultad de Veterinaria, 2006, Cáceres, España, Editors: D. Reina, J. Tovar, pp.277-326. Disponível em: https://www.researchgate.net/publication/247777715. Acesso em: 8 de setembro 2016.

35. Kaplan RM, Nielsen MK. An evidence-based approach to equine parasite control: itain't the 60s anymore. Equine Veterinary Education, Newmarket. 2010;22(6):306-316. Disponível em: http://dx.doi.org/10.1111/j.2042-3292.2010.00084.x. Acesso em: 8 de setembro de 2016.

36. Traversa D, Von Samson-Himmelstjerna G, _Demeler J, Milillo P, Schürmann S, Barnes H, Otranto D, Perrucci S, Di Regalbono AF, Beraldo P, Boeckh A, Cobb R. Anthelmintic resistance in cyathostomin populations from horse yards in Italy, UK and Germany. Parasites \& Vectors, London. 2009;2(2):1-7. Disponível em: https://doi.org/10.1186/1756-3305-2-S2-S2. Acesso em: 21 de agosto de 2016.

37. Mercadante A, Castro EA, Thomaz-Soccol V, Rompani JLA, Depra NM, Hennig L, Moraes FR. Ocorrência de resistência aos anti-helmínticos em equinos puro sangue, na região metropolitana de Curitiba. In: SEMINÁRIO BRASILEIRO DE PARASITOLOGIA VETERINÁRIA, 1997, Itapema. Revista Brasileira de Parasitologia Veterinária.1997,6:246.

38. Borges AF, Nakamura AY, Almeida GD, Cadamuro VHA. Eficácia de formulações anti-helmínticas comerciais em equinos no município de Douradina, Paraná. Ciência Animal Brasileira, Goiânia. 2010,11(3):618-622. Disponível em: https://www.researchgate.net/publication/47347992. Acesso em: 21 de agosto de 2016.

39. Cutolo AA, Dos Santos AT, Allegretti SM. Field study on the efficacy of an oral $2 \%$ ivermectin formulation in horses. Revista Brasileira de Parasitologia Veterinária, Jaboticabal. 2011,20(2):171-175. Disponível em: http://www.redalyc.org/articulo.oa?id=397841480015. Acesso em: 25 de agosto de 2016.

40. Felippelli G, Cruz BC, Gomes LV, Lopes WD, Teixeira WF, Maciel WG, Buzzulini C, Bichuette MA, Campos GP, Soares VE, Bergamasco PL, de Oliveira GP, da Costa AJ. Susceptibility of helminth species from horses against different chemical compounds in Brazil. Veterinary Parasitology. Amsterdam. 2015,212(34):232-238. Disponível em: https://doi.org/10.1016/j.vetpar.2015.07.041. Acesso em: 25 de agosto de 2016.

41. Dobrowolski EC, Slompo D, Carrasco AOT, Seki MC. Eficácia do praziquantel e da ivermectina em equinos infectados naturalmente com ciatostomíneos. Revista Acadêmica: Ciência Animal, Curitiba. 2016,14:75-81. Disponível em: http://dx.doi.org/10.7213/academica.14.2016.08. Acesso em: 25 de agosto de 2016.

42. Kaplan, RM. Anthelmintic resistance in nematodes of horses. Veterinary Research. 2002,33(5):491-507. 
Disponível em: https://doi.org/10.1051/vetres:2002035. Acesso em: 04 de setembro de 2016.

43. Reichmann P, Lisboa JAN, Balarin MRS, Pereira ABL. Valores hematológicos em equinos naturalmente infectados por estrongilídeos. Semina: Ciências Agrárias. 2001,22(2):179-181. Disponível em: http://dx.doi.org/10.5433/1679-0359.2001v22n2p179. Acesso em: 04 de setembro de 2016.

44. Lhamas CL, Duarte CA, Lübeck I, Silva MNG, Leite CT, Pereira EP, Roschildt K, Bernardon F. Influência do parasitismo intestinal sobre os parâmetros hematológicos e de líquido peritoneal em equinos de tração Arquivo Brasileiro de Medicina Veterinária e Zootecnia. 2015;67(2):381-390. Disponível em: http://dx.doi.org/10.1590/1678-7378. Acesso em: 05 setembro de 2016.

45. Sartori Filho R, Amarante AET, Oliveira MR. Efeito de medicações anti-helmínticas com ivermectin e fenbendazole em equinos: exames coprológicos e hematológicos. Revista Brasileira de Parasitologia Veterinária. 1993;2(1):61-64. Disponível em: http://cbpv.org.br/rbpv/documentos/211993/c2161_64.pdf. Acesso em: 12 setembro de 2016.

46. Duncan JL, Pirie HM. The pathogenesis of single experimental infections with Strongylus vulgaris in foals. Research in Veterinary Science. 1975;18(1):82-93. Disponível em: https://www.ncbi.nlm.nih.gov/pubmed/1118668. Acesso em: 14 setembro de 2016. 